\title{
11. SYMBOLIC POLE/ZERO ANALYSIS
}

\author{
Francisco V. Fernández \\ Dept. Electronics and Electromagnetism, University of Sevilla and IMSE-CNM, CSIC \\ Carlos Sánchez-López \\ IMSE-CNM, CSIC and University of Sevilla, Spain, and University of Tlaxcala, México \\ Rafael Castro-López \\ IMSE-CNM, CSIC and University of Sevilla \\ Elisenda Roca-Moreno \\ IMSE-CNM, CSIC and University of Sevilla
}

\begin{abstract}
Extraction of pole/zero expressions as a function of circuit parameters has traditionally been an essential tool for designers. In this Chapter, the main specific techniques for symbolic pole/zero extraction are described and their pros and cons are discussed. The application of the different techniques is illustrated with experimental results on practical circuits.
\end{abstract}

\section{Introduction}

Symbolic analysis tools automate the analysis, usually of linear (or linearized) circuits, in which all or part of the circuit parameters are kept in symbolic form. The output is usually in the form of a network function in the complex frequency variable:

$$
H(s)=\frac{\sum_{j=1}^{m} s^{j} f_{j}(x)}{\sum_{i=1}^{n} s^{i} g_{i}(x)}
$$

In addition to the network functions, e.g. magnitude or phased of the voltage gain of an amplifier, poles and zeros provide extremely valuable design knowledge. A direct extraction of symbolic poles and zeros from previously calculated network functions like (1) usually yields poor results. The first reason is that only second-order polynomials can be considered. Although analytical solutions for third-order and fourth-order polynomials exist, they are impractical in a symbolic analysis context. On the other hand, as Chapter 7 illustrates, the network function complexity grows exponentially with the circuit size. Therefore, the calculation of symbolic poles and zeros inherits this problem, even it worsens as a number of operations between network function coefficients has to be performed.

Therefore, the application of approximated extraction techniques together with the application of approximation techniques to the generation of symbolic expressions becomes mandatory. In this chapter, main reported techniques are discussed. In 
Section 2, the classical root splitting technique for approximated root extraction is introduced. This technique is combined together with simplification after generation techniques for reduction of expression complexity. As the plain application of simplification after generation techniques still limits dramatically the circuit size that can be analyzed, extraction techniques that make use of simplification before generation techniques (approximation of the network equations before they are symbolically solved) are discussed in Section 3. A more mature technique is introduced in Section 4, that uses the time-constant approach, enabling the introduction of simplification before and during generation techniques ${ }^{1}$.

\section{Classical root splitting technique}

Analytical calculation of poles and zeros from the symbolic numerator and denominator polynomials in (1) is theoretically limited to fourth-order polynomials, but practical use in the symbolic analysis context is limited to second-order polynomials. This implies that symbolic pole-zero extraction would be impossible except for the simplest circuits. To avoid this, common approximations performed in manual analysis like the root splitting technique were the first techniques applied for symbolic pole/zero extraction.

If we assume that the $n$ roots of the denominator in (1) are real, then the denominator can be written as

$$
D(s)=g_{0}\left(1-\frac{s}{p_{1}}\right)\left(1-\frac{s}{p_{2}}\right) \cdots\left(1-\frac{s}{p_{n}}\right)
$$

From the comparison with (1), it follows that:

$$
\begin{gathered}
g_{1}=g_{0} \sum_{i=1}^{n}\left(-\frac{1}{p_{i}}\right) \\
g_{2}=g_{0} \sum_{i=1}^{n} \sum_{j=i}^{n} \frac{1}{p_{i} p_{j}} \\
g_{3}=g_{0} \sum_{i=1}^{n} \sum_{j=i}^{n} \sum_{k=j}^{n}\left(-\frac{1}{p_{i} p_{j} p_{k}}\right)
\end{gathered}
$$

Under the assumption that the first pole is at a much lower frequency than the rest of poles:

\footnotetext{
1 See Chapter 7 for a detailed discussion of simplification before, during and after generation techniques.
} 


$$
\left\|p_{1}\right\| \ll\left\|p_{2}\right\|, \cdots,\left\|p_{n}\right\|
$$

the first equation in (3) can be approximated as:

$$
g_{1} \approx g_{0}\left(-\frac{1}{p_{1}}\right)
$$

and therefore:

$$
p_{1} \approx-\frac{g_{0}}{g_{1}}
$$

Under the assumption that the second pole is at a much lower frequency than higher order poles, the same procedure can also be applied resulting in:

$$
p_{2} \approx-\frac{g_{1}}{g_{2}}
$$

The same procedure can be applied iteratively for higher order poles. A similar procedure can also be applied if two complex poles (or two close real poles) are at a sufficiently lower frequency than other poles:

$$
\left\|p_{1}\right\| \approx\left\|p_{2}\right\| \text { and }\left\|p_{1}\right\| \ll\left\|p_{3}\right\|, \cdots,\left\|p_{n}\right\|
$$

Then, the pair of real or complex conjugate poles can be extracted from a secondorder polynomial:

$$
D(s) \approx g_{0}+g_{1} s+g_{2} s^{2}
$$

An analogous mechanism can be followed for the zeros of (1).

This technique was first implemented in [1],[2]. Since the complexity of symbolic network functions grows exponentially with the circuit size, the root splitting technique is not able to produce symbolic expressions of reasonable size for practical circuits by itself. Therefore, it must be applied on approximated functions, obtained from the application of simplification after generation (SAG) techniques ${ }^{2}$. In these techniques, the exact symbolic network function is first generated and, afterwards, the least significant terms are pruned while some error criterion is met. For instance, given any network function coefficient in (1), formulated as a sum-of-product of symbolic parameters:

\footnotetext{
${ }^{2}$ Refer to Chapter 7 for a detailed description of these techniques.
} 


$$
h_{k}(x)=\sum_{l=1}^{L} h_{k l}(x)
$$

the approaches in [2],[3] eliminate the $P$ least significant terms while the following condition is satisfied:

$$
\frac{\sum_{l=1}^{P}\left|h_{k l}\left(x_{\boldsymbol{o}}\right)\right|}{\sum_{l=1}^{L}\left|h_{k l}\left(\boldsymbol{x}_{\boldsymbol{o}}\right)\right|} \leq \varepsilon
$$

where $\varepsilon$ is a user-defined accuracy margin and $\boldsymbol{x}_{\boldsymbol{o}}$ is the point of the design parameter space selected for the evaluation of the approximation error.

If the poles and zeros are to be extracted from the simplified network function, and in order to keep the root positions unchanged, the same error threshold $\varepsilon$ can be applied for each coefficient of the network function. However, this may lead to important errors in pole and zero locations due to the very nature of the simplification process. This process consists in eliminating individual symbolic terms of each coefficient and, therefore, the estimated coefficient value varies at discrete steps. Then, the real error in each polynomial coefficient will be different and this may yield very significant shifts of root locations. To avoid this problem, the approach in [1] eliminates the least significant terms in each coefficient incrementally. A small value of $\varepsilon$ is used initially and it is increased by $\Delta \varepsilon$ in each iteration. At each iteration, poles and zeros are monitored. It the root shifts are larger than a predefined value, symbolic approximation is stopped. A possible problem is that roots cannot be easily traced when discrete approximations are applied, e.g. elimination of certain terms may yield unexpected root shifts with difficult correlation with the previous location.

As an example of application let us consider the cascode amplifier in Fig. 1(a) where the small-signal model in Fig. 1(b) is used for the MOS transistors ${ }^{3}$. The application of the technique in [1] provides symbolic expressions for the first two poles and the first zero:

\footnotetext{
${ }^{3}$ The experimental results shown in this chapter are intended for illustrative purposes and coarse comparison. Accurate comparison is infeasible as the implementations of most approaches are not freely or commercially available and we are restricted to the results available in the literature. In many cases, the different approaches have been tested on different circuits. Even in those cases that have been tested on the same circuit, the results may not be comparable as they may be influenced by the extraction technique itself but by the nominal value of the symbolic parameters (around which the simplification is performed) and by the configuration settings of each approach. In most cases, a complete information has not been disclosed.
} 


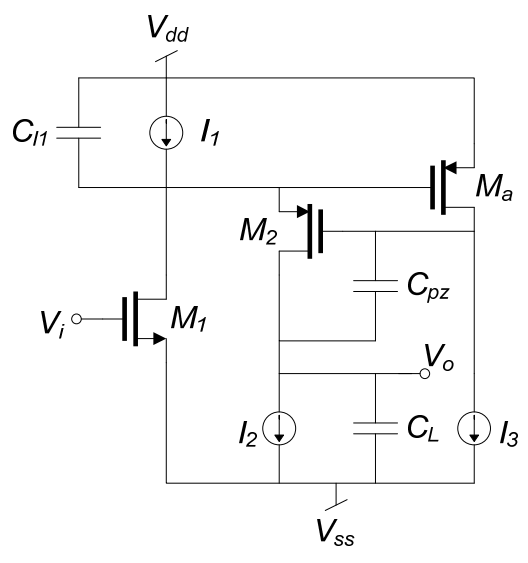

(a)

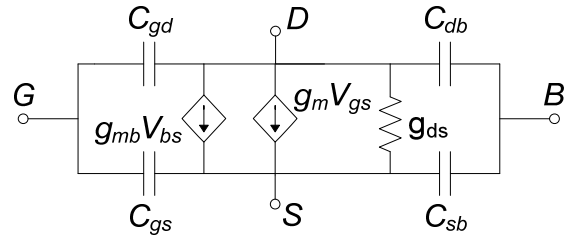

(b)

Figure 1: (a) Active feedforward compensated cascode amplifier and (b) small-signal MOS transistor model.

$$
\begin{gathered}
p_{1}=-\frac{g_{d s 1} g_{d s 2} g_{d s a}}{g_{m 2} g_{m a}\left(C_{L}+C_{g d 2}+C_{p z}\right)} \\
p_{2}=-\frac{g_{m a}\left(C_{L}+C_{g d 2}+C_{p z}\right)}{\left(C_{g d 2}+C_{p z}\right)\left(C_{L}+C_{d b 1}+C_{g d 1}+C_{l 1}\right)} \\
z_{1}=-\frac{g_{m a}}{\left(C_{g d 2}+C_{p z}\right)}
\end{gathered}
$$

Valuable information can be extracted from (12), like the value of the capacitor $C_{p z}$ that cancels the pole-zero pair:

$$
C_{p z}=C_{d b 1}+C_{g d 1}+C_{l 1}-C_{g d 2}
$$

A posterior technique tried to reduce the error in the pole-zero locations by applying one or more Newton-Raphson iterations on the locations predicted by the root splitting technique [4]. A pole/zero at the $n$th iteration of the Newton-Raphson algorithm is given by:

$$
s_{n+1}=s_{n}-\frac{F\left(s_{n}\right)}{F^{\prime}\left(s_{n}\right)}
$$

This involves the symbolic computation of the quotient of a polynomial (numerator or denominator of the network function evaluated at the symbolic solution of the root obtained in the previous iteration) and its derivative with respect to the complex frequency variable $s$. In general, the price to pay is a significant increase of the complexity of the symbolic expressions, making the interpretability of the symbolic results more difficult. 
The major problems of the root splitting technique applied to symbolic pole/zero extraction are:

a) The extraction technique shares the limitations of simplification after generation techniques. Remind from Chapter 7 that simplification after generation techniques require the previous generation of the exact network function. The exponential growth of expression complexity with the circuit size limits the maximum analyzable circuit size to a few tens of elements (circuit elements after semiconductor devices have been replaced by their small-signal models).

b) The approximation may yield significant pole/zero errors. Techniques that avoid this, like [1], may lead to significant increase of expression complexity as the simplification may be stopped prematurely.

\section{SBG-based techniques}

Another group of techniques are based on the application of simplification before generation (SBG) techniques on a matrix formulation of the network equations. For generic linear circuits the Modified Nodal Analysis formulation offers a suitable procedure to build a set of linear equations in the Laplace domain. Besides, this method allows a clear splitting between the static and dynamic components of the circuit, that is,

$$
\boldsymbol{Y}_{M N A}=\boldsymbol{G}+s \boldsymbol{C}
$$

where the matrix $\boldsymbol{G}$ contains the static elements (e.g., conductances, controlled sources) and the matrix $\boldsymbol{C}$ contains the dynamic elements (capacitors and inductors). To ease the subsequent formulation and implementation, inductors are considered in impedance form [5].

Poles are defined as those complex values that lead to the following set of homogeneous equations:

$$
(G+s C) x=0
$$

or equivalently:

$$
G x=-s C x
$$

Solution of (17) corresponds to the well-known generalized eigenvalue problem. The usual mechanism for the numerical solution of (17) is the QZ algorithm [6]. This algorithm can be used for the numerical calculation of poles and zeros but its iterative nature prevents its use for symbolic pole/zero extraction.

To overcome the circuit size limitations of symbolic pole/zero extraction methodologies based on simplification after generation techniques, new approaches 
have been reported in [7]-[9] whose distinctive feature is the introduction of simplification before generation techniques.

The approach in [7] is based on a simplification before generation technique reported in [10]. The key step is the elimination of device parameters from the cofactors of the nodal matrix if the error induced is below a given error margin. The error is evaluated at a few samples (usually three points per decade) of the frequency range of interest. The frequency range has to be selected to isolate a small cluster of poles or zeros, resembling the classical root splitting technique. Then, the determinant dimension is tried to be reduced by factoring out rows and columns with a single symbol or nonzero entry and performing row and common operations that reduce the number of symbols or non-zero entries. Determinant expansion is performed trying to keep a factorized form that can ease extraction of poles and zeros although it is not clear how the factorization can be performed systematically to yield the appropriate form for pole/zero extraction.

The application of this technique to the Miller op amp in Fig. 2 in the frequency range from dc to $1 \mathrm{KHz}$ allows to obtain the following expression for the first pole [7]:

$$
p_{1}=-\frac{\left(g_{d s 2}+g_{d s 4}\right)\left(g_{d s 6}+g_{d s 7}\right)}{g_{m 6} C_{c}}
$$

The subsequent application to the frequency range which goes from $7 \mathrm{MHz}$ to $70 \mathrm{MHz}$ provides an easy factorization of three symbolic poles [7]:

$$
\begin{gathered}
p_{2}=-\frac{C_{c} g_{m 6}+\left(g_{d s 6}+g_{d s 7}\right)\left(C_{g b 6}+C_{g d 2}+C_{g d 4}+C_{g s 6}\right)}{\left(C_{c}+C_{g d 7}\right)\left(C_{g b 6}+C_{g d 2}+C_{g d 4}+C_{g s 6}\right)} \\
p_{3}=-\frac{\left(g_{m 1}+g_{m 2}\right)}{\left(C_{g b 1}+C_{g b 2}+C_{g s 1}+C_{g s 2}\right)} \\
p_{4}=-\frac{g_{m 3}}{\left(C_{g b 3}+C_{g b 4}+C_{g d 1}+C_{g d 4}+C_{g s 3}+C_{g s 4}\right)}
\end{gathered}
$$

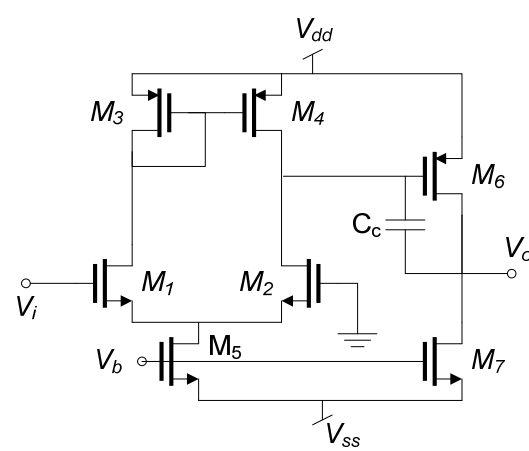

(a)

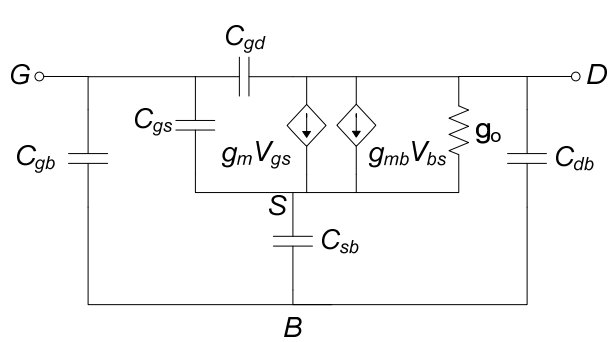

(b)

Figure 2: (a) Two-stage CMOS Miller opamp and (b) small-signal MOS transistor model. 
However, pole $p_{3}$ is not a valid pole as it cancels with an analogous zero at the numerator [7]. This is probably due to the fact that the evaluation of the errors introduced by device parameter elimination is performed in the corresponding cofactor and not in the complete network function.

On the other hand, there is no clear procedure to select the frequency range and the number of samples that provides the right expression for easier root factorization. Small root shifts may produce very significant magnitude/phase errors depending on the root location in the complex frequency plane, therefore stopping the simplification process much before than needed, hence, yielding very complex symbolic expressions. On the contrary, important root displacements may keep undetected by magnitude/phase error criteria if the number of samples is not appropriate. The problem palliates by increasing the number of samples at the price of additional computation time.

The approach in [8] also performs a simplification before generation technique and tries to avoid the problems of simplification techniques based on magnitude/phase error control. In this case, a single root is selected a priori and the errors induced by parameter eliminations are not evaluated in the cofactors but in the root itself. This could be calculated by the application of the QZ algorithm. However, this algorithm presents two drawbacks in this application. First, although we are interested in a single root, the algorithm calculates the complete root spectrum. And second, the computational cost of the algorithm is relatively high, making inefficient its application for each device parameter elimination that is tried. To avoid this problem, [8] uses the sensitivity of the root to each device parameter, assuming that the sensitivity is directly related to the root shift caused by cancelling that device parameter. This becomes a weakness of this approach as a small local sensitivity is not a guarantee for a small large-change sensitivity represented by the device parameter elimination.

Once all device parameters with limited impact on pole/zero locations have been eliminated, the determinant is expanded and a polynomial in the complex frequency is obtained. Simplification-after-generation techniques are applied in the coefficients of this polynomial. Hopefully, the polynomial degree is low enough for symbolic extraction of the root. Again additional simplification after generation is tried in the resulting symbolic expression.

Another approach based on simplification before generation techniques to extract poles and zeros was reported in [9]. As the previous one it is based on the approximation of the matrices in (16) for a selected eigenvalue by ranking the eigenvalue shifts induced by different device parameter eliminations and performing the least significant device prunings while some error criterion in the eigenvalue shift is met. The eigenvalue shift is obtained from a linear prediction formula derived from a Taylor series approximation of the generalized eigenvalue problem, similar to the sensitivity analysis above, yielding a ranking of candidate parameter eliminations.

To solve the problem of underestimation of real errors due to significantly bigger large-change sensitivity, this approach estimates pole-zero shifts for each parameter 
elimination that is performed. For the same reasons above, error tracking by the application of the QZ algorithm is not efficient enough. Therefore, an error tracking by iterative eigenvalue improvement process is applied. If the eigenvalue shift induced by a device parameter elimination is small, we can assume that the nominal value of the eigenvalue is a good initial guess for an iterative calculation process that converges to the perturbed eigenvalue. If the method fails to converge, we can assume that the eigenvalue shift is too large.

Like in previous methods that monitor pole/zero shifts there is a risk of a bad identification of an eigenvalue with its perturbed counterpart. To avoid this, a very significant improvement is introduced in [9], where a modal assurance criterion (MAC) is defined, that measures the correlation of two eigenvectors and that is given as [9]:

$$
\operatorname{MAC}\left(\boldsymbol{u}, \boldsymbol{u}^{*}\right)=\frac{\left|\boldsymbol{u}^{H} \boldsymbol{u}^{*}\right|^{2}}{\left(\boldsymbol{u}^{H} \boldsymbol{u}\right)\left(\boldsymbol{u}^{* H} \boldsymbol{u}^{*}\right)}
$$

where $\boldsymbol{u}$ is the eigenvector corresponding to the selected eigenvalue of the original system in (16) and $\boldsymbol{u}^{*}$ is the eigenvector of the perturbed system.

If a perturbed eigenvector corresponds to the original one, they must be closely correlated and therefore, the modal assurance criterion must be close to 1 . By the contrary, if the modal assurance criterion is much smaller, it means that the eigenvectors do not correspond and the approximation is not valid, even if they are numerically very close.

The determinant of the matrix resulting after the simplification before generation technique is expanded. Hopefully again, a low-order polynomial is obtained from which the desired root can be extracted. Simplification after generation techniques can be applied for further formula simplification. Robust simplification during generation techniques like those reviewed in Chapter 7, are not in general applicable as they cannot be applied on matrix formulations.

As illustrative examples, several experimental results have been reported with this approach, like the first two poles of the uA741 operational amplifier in Fig. 3.

By applying simplification before generation techniques based on magnitude/phase error control the first pole is obtained:

$$
p_{1}=-\frac{\left(r_{o 13 B}+r_{o 17}\right)\left(R_{9} r_{o 4}+g_{m 17} R_{8} r_{\pi 17}\left(r_{o 4}+g_{m 16} R_{9} r_{\pi 16}\right)\right.}{C_{1} g_{m 16} g_{m 17} R_{9} r_{o 13 B} r_{o 17} r_{o 4} r_{\pi 16} r_{\pi 17}}
$$

However, the second pole cannot be obtained with this technique due to the existence of a close zero. However, the approach in [9] allows obtaining a symbolic expression for the second pole: 


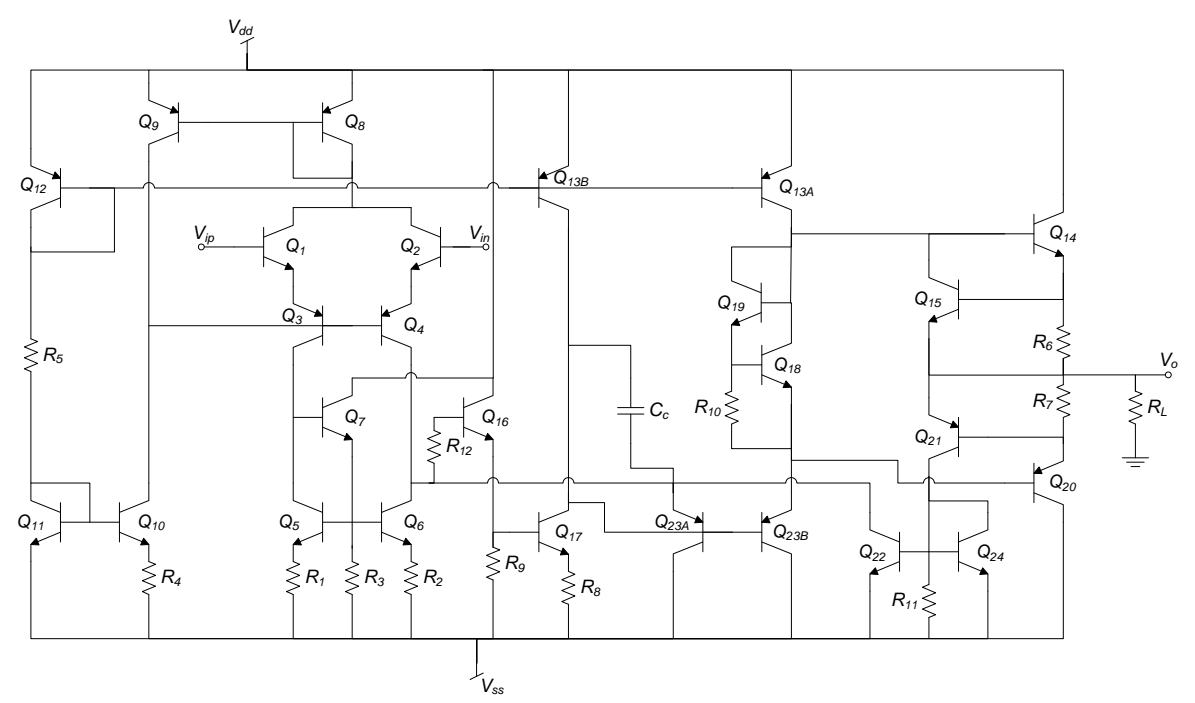

(a)

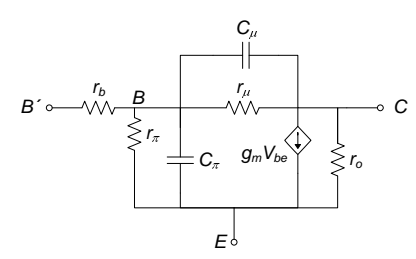

(b)

Figure 3: (a) $\mu$ A741 operational amplifier and (b) small-signal bipolar transistor model.

$$
p_{2}=-\frac{1}{R_{11}\left(C_{\mu 21}+C_{\mu 22}+C_{\pi 22}+C_{\pi 24}\right)}
$$

The two-stage amplifier in Fig. 4 is used as a second example. An error bound of 5\% in pole-zero shifts allows to obtain symbolic expressions for the first two poles and the first zero:

$$
\begin{gathered}
p_{1}=-\frac{\left(g_{d s 2}+g_{d s 4}\right)\left(g_{d s 6}+g_{d s 7}\right)}{g_{m 6} C_{c}+\left(g_{d s 2}+g_{d s 4}\right) C_{L}} \\
p_{2}=-\frac{g_{d s 9}\left(C_{c} C_{g s 6}+C_{c} C_{L}+C_{L} C_{g s 6}\right)-\sqrt{g_{d s 9}^{2}\left(C_{c} C_{g s 6}+C_{c} C_{L}+C_{L} C_{g s 6}\right)^{2}-4 C_{c}^{2} C_{L} C_{g s 6} g_{d s 9} g_{m 6}}}{2 C_{c} C_{L} C_{g s 6}} \\
z_{1}=\frac{g_{d s 9} g_{m 6}}{C_{c}\left(g_{d s 9}-g_{m 6}\right)}
\end{gathered}
$$




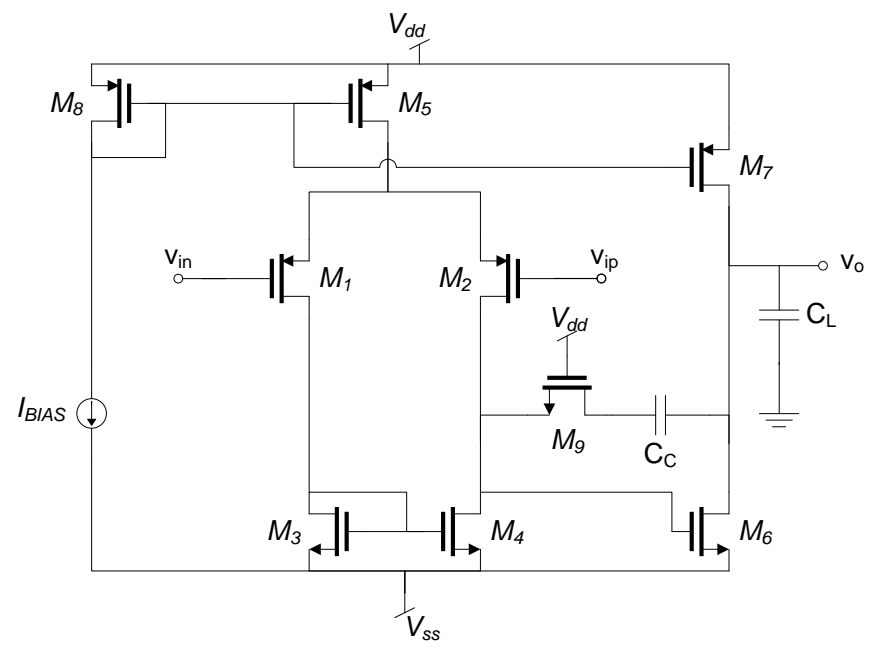

Figure 4: Two-stage Miller amplifier.

\section{The time-constant approach}

\subsection{Haley's modification-decomposition method}

To arrive at a solution for the symbolic pole-zero extraction, [11] proposes an approach inspired on Haley's modification-decomposition method [12],[13]. Haley's modification-decomposition method starts by premultiplying (17) by $\boldsymbol{G}^{-1}$ :

$$
\boldsymbol{x}=-s \boldsymbol{G}^{-1} \boldsymbol{C} \boldsymbol{X}
$$

Assuming that the circuit contains $M$ capacitors, matrix $\boldsymbol{C}$ can be expressed as:

$$
C=\mathfrak{I} C_{d} \mathfrak{I}^{T}
$$

where $\boldsymbol{C}_{\boldsymbol{d}}$ is the following diagonal matrix:

$$
\boldsymbol{C}_{d}=\left[\begin{array}{cccc}
C_{1} & 0 & \cdots & 0 \\
0 & C_{2} & \cdots & 0 \\
\vdots & \vdots & & \vdots \\
0 & 0 & \cdots & C_{M}
\end{array}\right]
$$

And the capacitor incidence matrix $\mathfrak{I}$ is given by:

$$
\mathfrak{I}=\left[\begin{array}{llll}
\boldsymbol{q}_{1} & \boldsymbol{q}_{2} & \cdots & \boldsymbol{q}_{M}
\end{array}\right]
$$

where the vector $\boldsymbol{q}_{k}$ corresponding to the $k$-th capacitor connected between nodes $i$ and $j$ : 


$$
\boldsymbol{q}_{k}=\left[\begin{array}{lllllll}
0 & \cdots & 1 & \cdots & -1 & \cdots & 0
\end{array}\right]^{T}
$$

By premultiplying (24) by $\mathfrak{J}^{T}$ and replacing (25) it yields:

$$
\mathfrak{I}^{T} \boldsymbol{x}=-s \mathfrak{I}^{T} \boldsymbol{G}^{-1} \mathfrak{I} \boldsymbol{C}_{\boldsymbol{d}} \mathfrak{I}^{T} \boldsymbol{X}
$$

By redefining its components:

$$
\begin{gathered}
\boldsymbol{x}_{\boldsymbol{M}}=\mathfrak{I}^{T} \boldsymbol{X} \\
\boldsymbol{R}=\mathfrak{I}^{T} \boldsymbol{G}^{-1} \mathfrak{J} \\
\boldsymbol{T}=\boldsymbol{R} \boldsymbol{C}_{\boldsymbol{d}}
\end{gathered}
$$

Eq. (29) becomes:

$$
\boldsymbol{X}_{M}=-s \boldsymbol{T} \boldsymbol{x}_{M}
$$

By performing the following variable change:

$$
\tau=-\frac{1}{S}
$$

(31) becomes:

$$
(\boldsymbol{T}-\tau \mathbf{1}) \boldsymbol{x}_{M}=\mathbf{0}
$$

This is called a modification of matrix $\boldsymbol{G}$ and its effect is the transformation of an $\mathrm{N}$ dimensional generalized eigenvalue problem into an $M$-dimensional standard eigenvalue problem, whose eigenvalue spectrum can be related to that of the generalized eigenvalue problem by using (32).

As reported in [12], an important advantage of this transformation is that the numerical calculation of the roots can be performed by using the QR algorithm, faster and more stable than the QZ algorithm.

However, the biggest advantage of this formulation in the symbolic context, as will be shown in the following, is the simple structure of the matrix $\boldsymbol{T}$ that provides valuable physical insight on the dynamic circuit behavior. Moreover, each entry of the matrix $\boldsymbol{T}$ can be symbolically calculated very efficiently, being the only approach that allows introducing both simplification before and during generation techniques, enabling the highest accuracy with the smallest expression complexity. 


\subsection{The time-constant matrix}

Let us consider the structure of matrix $\boldsymbol{T}$ in (30). The element located in row $\alpha$ and column $\beta$ is given by:

$$
T_{\alpha \beta}=\boldsymbol{q}_{\alpha}^{T} \boldsymbol{G}^{-1} \boldsymbol{q}_{\beta} C_{\alpha}
$$

In this equation we can identify a vector

$$
\boldsymbol{y}=\boldsymbol{G}^{-1} \boldsymbol{q}_{\beta}
$$

that represents the nodal voltages when the resistive part of the circuit is excited by a single source placed at the port defined by capacitor $C_{\beta}$. Then, the linear combination

$$
\boldsymbol{q}_{\alpha}^{T} \boldsymbol{y}
$$

represents the voltage across the port defined by capacitor $C_{\alpha}$. Therefore, the matrix element can be interpreted as the transresistance from port to port multiplied by capacitor $C_{\alpha}$ and matrix $\boldsymbol{T}$ becomes:

$$
\boldsymbol{T}=\left[\begin{array}{cccc}
R_{11} C_{1} & R_{12} C_{2} & \cdots & R_{1 M} C_{M} \\
R_{21} C_{1} & R_{22} C_{2} & \cdots & R_{2 M} C_{M} \\
\vdots & \vdots & \vdots & \vdots \\
R_{M 1} C_{1} & R_{M 2} C_{2} & \cdots & R_{M M} C_{M}
\end{array}\right]
$$

It becomes clear now why matrix $\boldsymbol{T}$ is known as the time-constant matrix (or RCmatrix). Its elements are RC products that can be computed directly from the circuit.

Valuable information can be obtained from this matrix. For instance, classical hand analysis techniques formulate the poles of circuits as the inverse of the capacitance at each circuit node multiplied by the impedance seen at that node. Matrix $\boldsymbol{T}$ gives a more precise definition and the conditions under which it is valid. A pole can be considered to be associated to one node if the (trans)resistances of the other ports to that port are negligible.

\subsection{Approximate root equations}

The sum of products of the eigenvalues of matrix $\boldsymbol{T}$ are related to its $k$-th trace by:

$$
\sum_{i_{1}<i_{2}<\cdots<i_{k}} \tau_{i_{1}} \cdot \tau_{i_{2}} \cdot \ldots \cdot \tau_{i_{k}}=(-1)^{k} T_{k}
$$

where the $k$-th trace of matrix $\boldsymbol{T}$ is defined as: 


$$
T_{k}=\sum_{\alpha_{1}<\cdots<\alpha_{k}}\left[\begin{array}{cccc}
R_{\alpha_{1} \alpha_{1}} & R_{\alpha_{1} \alpha_{2}} & \cdots & R_{\alpha_{1} \alpha_{k}} \\
R_{\alpha_{2} \alpha_{1}} & R_{\alpha_{2} \alpha_{2}} & \cdots & R_{\alpha_{2} \alpha_{k}} \\
\vdots & \vdots & \vdots & \vdots \\
R_{\alpha_{k} \alpha_{1}} & R_{\alpha_{k} \alpha_{2}} & \cdots & R_{\alpha_{k} \alpha_{k}}
\end{array}\right] C_{\alpha_{1}} \cdots C_{\alpha_{k}}
$$

Let us consider the equations relating eigenvalues to the first two traces:

$$
\begin{gathered}
\sum_{i=1}^{M} \tau_{i}=-T_{1} \\
\sum_{i=1}^{M-1} \sum_{j=i+1}^{M} \tau_{i} \cdot \tau_{j}=T_{2}
\end{gathered}
$$

If the first two poles are sufficiently separated, i.e., the following two conditions are satisfied:

$$
\begin{gathered}
\tau_{1}+\tau_{2} \gg \sum_{i>2} \tau_{i} \\
\tau_{1} \cdot \tau_{2} \gg \sum_{i>2} \sum_{j>i} \tau_{i} \cdot \tau_{j}
\end{gathered}
$$

the following set of equations can be approximated

$$
\begin{aligned}
\tau_{1}+\tau_{2} & \approx-T_{1} \\
\tau_{1} \cdot \tau_{2} & \approx T_{2}
\end{aligned}
$$

If these two poles are close or constitute a complex conjugate pair, the following expression is easily obtained for the poles:

$$
p_{1,2} \approx-\frac{1}{2} \cdot \frac{T_{1}}{T_{2}} \cdot\left(1 \pm \sqrt{1-4 \cdot \frac{T_{2}}{T_{1}^{2}}}\right.
$$

However, if the two poles are splitted enough i.e. , a further approximation can be applied and the following expressions for $p_{1}$ and $p_{2}$ are obtained:

$$
\begin{aligned}
& p_{1} \approx-\frac{1}{T_{1}} \\
& p_{1} \approx-\frac{T_{1}}{T_{2}}
\end{aligned}
$$

The same approach can be applied recursively to higher frequency poles. Let us assume one port with a large time constant. This means that this pole remains practically uncharged for high-frequency signals, that is, it behaves like a shortcircuit. Therefore, the circuit resulting after the contraction of the capacitor associated 
to the port with the highest time-constant will have approximately the same higher frequency poles that the original one. This transformation yields a shift towards zero of the dominant pole whereas the remaining ones are kept in approximately the same locations. Therefore, once the contraction has been performed, the same extraction procedure can be applied to higher frequency poles.

\subsection{Symbolic calculation of RC factors}

Equations (43) and (44) approximate the pole positions as a function of the first two traces, given as:

$$
\begin{gathered}
T_{1}=\sum_{\alpha=1}^{M} R_{\alpha \alpha} \cdot C_{\alpha} \\
T_{2}=\sum_{\alpha=1}^{M-1} \sum_{\beta=\alpha+1}^{M}\left[\begin{array}{ll}
R_{\alpha \alpha} & R_{\alpha \beta} \\
R_{\beta \alpha} & R_{\beta \beta}
\end{array}\right] \cdot C_{\alpha} \cdot C_{\beta}
\end{gathered}
$$

Then, approximate expressions for these two traces are obtained in several steps. First, a simplification before generation step is applied for the circuit capacitors, by eliminating the capacitors that have an influence in $T_{1}$ and $T_{2}$ such that their impact on each pole position is below an error threshold. The effect of this simplification before generation step is that the number of addends in (45) is significantly reduced and only a reduced number of (trans)resistances has to be calculated.

In a second step, a circuit is considered for the calculation of each (trans)resistance in (45). Each of these circuits is a purely resistive circuit obtained from that resulting from the previous step by removing all capacitors and applying the appropriate excitation source for each (trans)resistance. For illustration's sake, Fig. 5 shows the transformations on the original circuit for the calculation of the transresistances of a

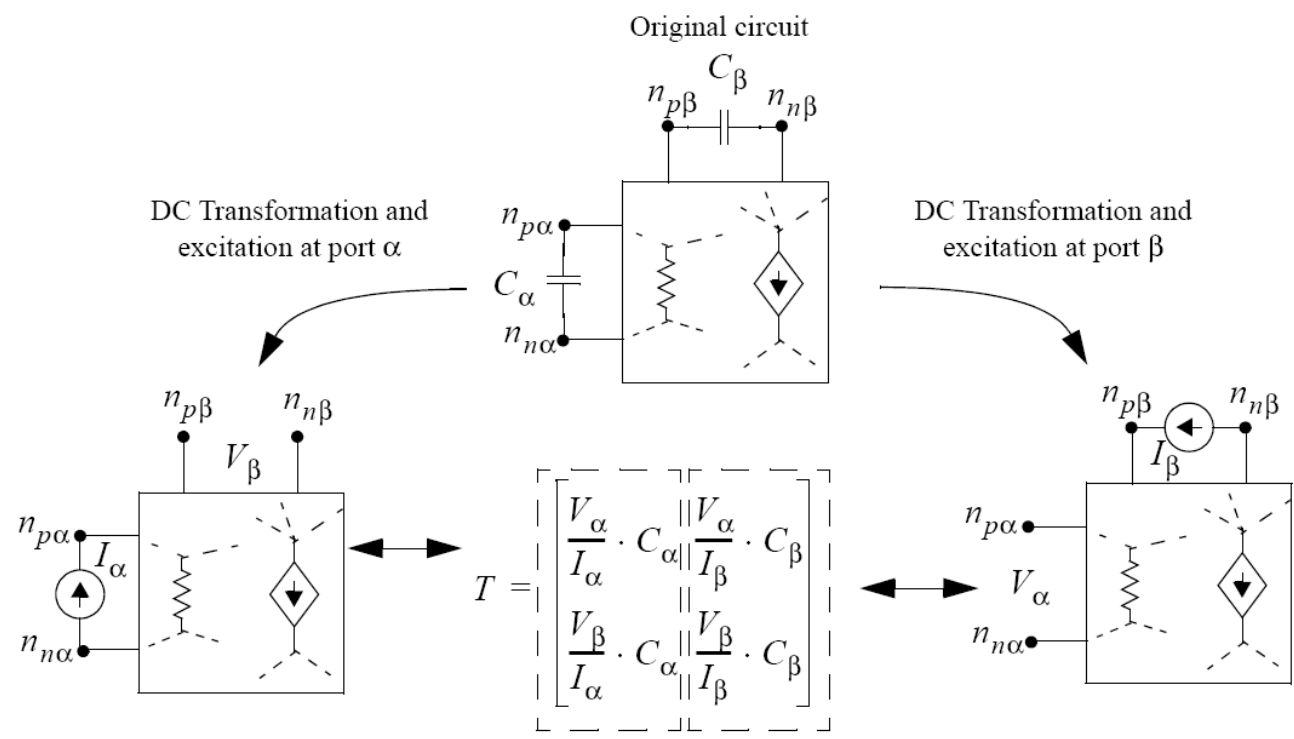

Figure 5: Illustrating circuit transformation for calculation of (trans)resistances. 
circuit with two capacitors $C_{\alpha}$ and $C_{\beta}$. These resistive circuits can still be relatively complex and therefore also suffer from the exponential growth of symbolic results with the circuit size. Therefore, first a simplification before generation technique is applied. For reasons that will become clear below, the SBG technique works at the circuit level, by contracting nodes and eliminating devices whose contribution to the pole position is negligible. A separate circuit is considered for each (trans)resistance, so that the simplest circuit for each of them can be obtained.

Then, a simplification during generation technique is applied to get the dominant terms of each (trans)resistance. The simplification during generation technique applied is based on the two-graph approach and is able to generate symbolic terms in decreasing order of magnitude one-by-one. Initially, the dominant term of each (trans)resistance is generated and queued. The dominant term of each trace is considered and that with the largest influence on the pole or poles considered is selected for the symbolic pole expression. The following term for the (trans)resistance that supplied the selected symbolic term is then generated.

If the errors specs are not met, the next term in the list has to be added to $T_{1}$ or to $T_{2}$. At each iteration two ratios are used to decide if the next term in $T_{1}$ or that in $T_{2}$ should be collected. Assuming that $F$ terms have already been added into $T_{1}$ and $G$ terms into $T_{2}$, the two ratios are:

$$
\begin{gathered}
r_{1}=\frac{\sum_{k=1}^{F} R_{\alpha \alpha}^{(k)} \cdot C_{\alpha}^{(k)}}{\sum_{i=1}^{M} R_{i i} \cdot C_{i}} \\
r_{2}=\frac{\sum_{k=1}^{G}\left[\begin{array}{cc}
R_{\alpha \alpha}^{(k)} & R_{\alpha \beta}^{(k)} \\
R_{\beta \alpha}^{(k)} & R_{\beta \beta}^{(k)}
\end{array}\right] \cdot C_{\alpha}^{(k)} \cdot C_{\beta}^{(k)}}{\sum_{i=1}^{M-1} \sum_{j=i+1}^{M}\left[\begin{array}{ll}
R_{i i} & R_{i j} \\
R_{j i} & R_{j j}
\end{array}\right] \cdot C_{i} \cdot C_{k}}
\end{gathered}
$$

These equations represent the ratios between the terms added to $T_{1}$ and $T_{2}$ up to that iteration and the exact values given by the equations in (45). The next term in the trace with the smallest ratio is added and the errors specs checked again. The generation procedure continues until the error specs are met.

\subsection{Extraction of zeros}

The modification-decomposition method can also be applied to the zero extraction problem by performing some modifications on the time-constants matrix, leading to a modified time-constants matrix, from which the numerical zero spectrum can be computed by means of the QR algorithm. However, unlike the time-constants matrix, although the elements of the modified time-constants matrix are dimensionally RC products, they cannot be easily interpreted in terms of circuit (trans)resistances and capacitors. Therefore, the approximated formulas cannot be directly applied. 
A solution arises from the application of feedback systems theory. Let us consider the system in Fig. 6, where $H(s)$ corresponds to the transfer function of the system whose zeros are to be obtained. The transfer function of the complete feedback system is:

$$
H_{f b}(s)=\frac{k \cdot D(s)}{D(s)+k \cdot N(s)}
$$

If $k \rightarrow \infty$, then (47) becomes:

$$
H_{f b}(s)=\frac{D(s)}{N(s)}
$$

Therefore, the poles of the feedback system correspond to the zeros of the original one.

Dealing with the poles of this feedback system is not a complex task. The forward

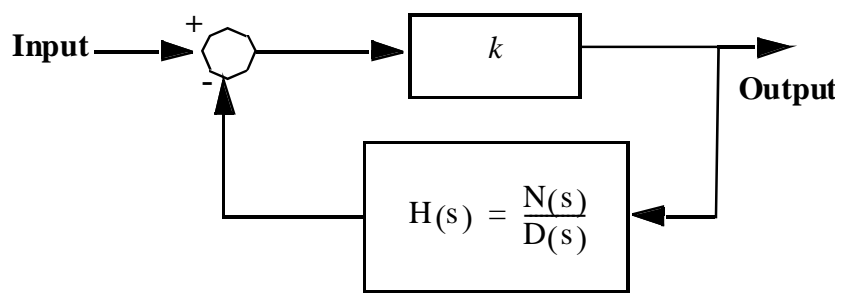

Figure 6: Block diagram.

gain $k$ does not depend on the complex frequency variable. Therefore, it contributes to the different (trans)resistances of the matrix $\boldsymbol{T}$. As stated above, simplification before and during generation techniques are applied to the different (trans)resistances. Simplification before generation techniques are applied normally but the element corresponding to the forward gain $k$ is preserved as the application of $k \rightarrow \infty$ prevents its elimination. The simplification during generation technique used is based on the enumeration of common spanning trees in the two-graph approach in decreasing order of magnitude. Applying $k \rightarrow \infty$ reduces to imposing that the element corresponding to $k$ must belong to the spanning tree ${ }^{4}$.

\subsection{Experimental results}

As an application example let us consider the uA741 operational amplifier in Fig. 4. The approach above provides the following expressions for the first two poles and the first zero:

\footnotetext{
${ }^{4}$ Refer to Chapter 7 for detailed descriptions of simplification before and during generation techniques.
} 


$$
\begin{gathered}
p_{1}=-\frac{g_{m 1} g_{m 3}+g_{m 1} g_{m 4}+g_{m 3} g_{m 4}}{C_{c} g_{m 1} g_{m 4} g_{m 16} g_{m 17} r_{o 13 B} r_{o 17} r_{\pi 16} r_{\pi 17}} \\
p_{2}=-\frac{g_{m 1} g_{m 3}+g_{m 1} g_{m 4}}{R_{11}\left(C_{\pi 22}+C_{\pi 24}\right)\left(g_{m 1} g_{m 3}+g_{m 1} g_{m 4}+g_{m 3} g_{m 4}\right)} \\
Z_{1}=-\frac{g_{m 10}\left(g_{m 5}+g_{m 6}\right) R_{4}}{R_{11}\left[\left(1+g_{m 10} R_{4}\right)\left(g_{m 5}+g_{m 6}+g_{m 5} g_{m 6}\left(R_{1}+R_{2}\right)\right]\left(C_{\pi 22}+C_{\pi 24}\right)\right.}
\end{gathered}
$$

If we now consider the BiCMOS operational amplifier in Fig. 7, the approach provides symbolic expressions for the four poles:

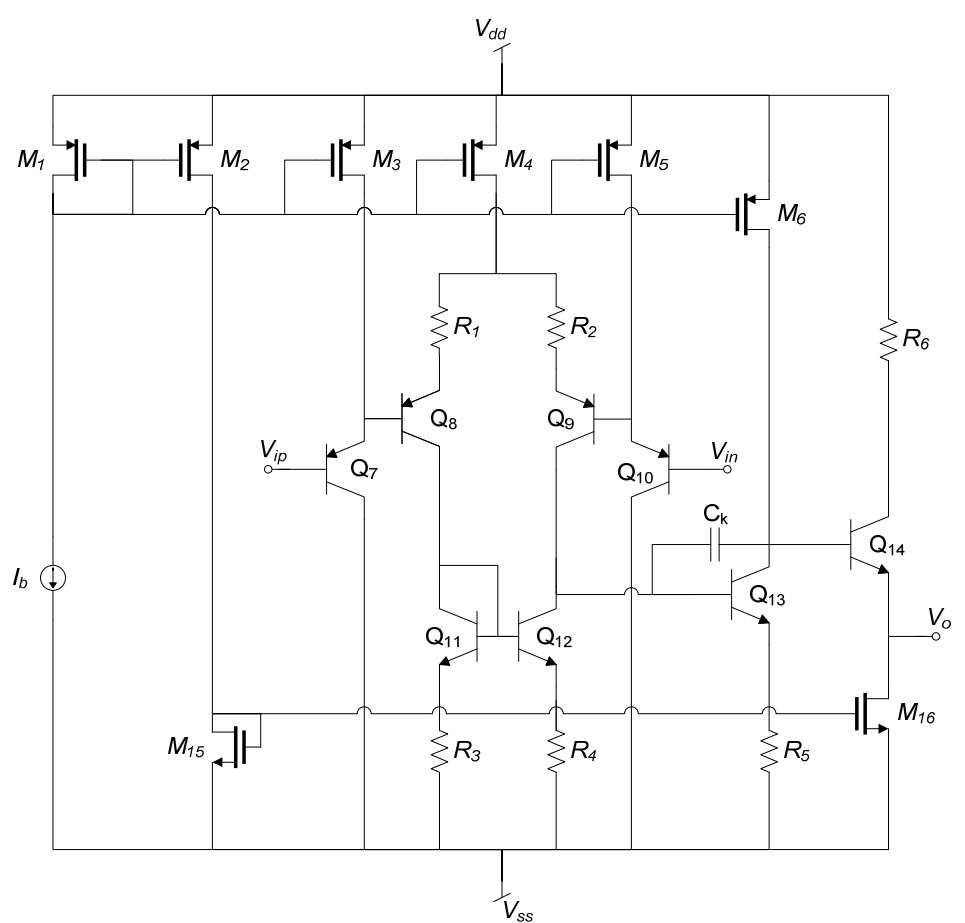

(a)

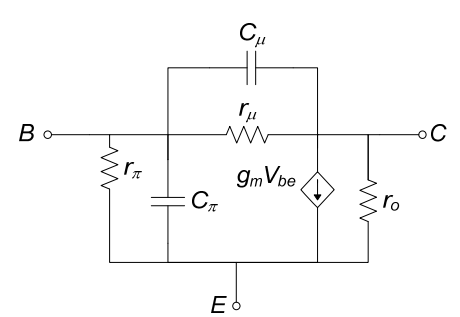

(b)

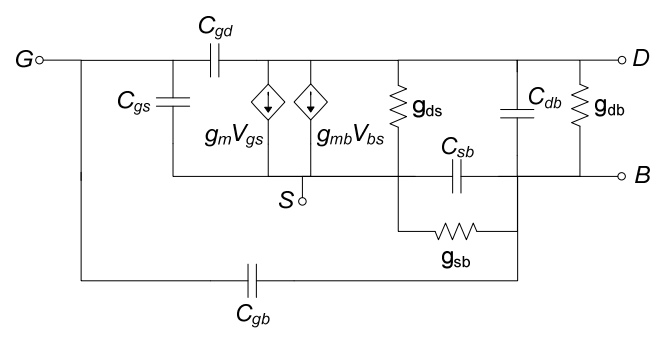

(c)

Figure 7: (a) BiCMOS operational amplifier; (b) bipolar transistor model; and (c) MOS transistor model. 


$$
\begin{gathered}
p_{1}=-\frac{g_{d s 6}\left(R_{1}+R_{2}\right)\left[g_{m 12} R_{4}\left(g_{m 1}+g_{d s 1}\right)+g_{m 1}\right]}{r_{\pi 13} g_{m 1} g_{m 12} g_{m 13} R_{1} R_{4}\left(C_{k}+C_{\mu 13}\right)} \\
p_{2}=-\frac{g_{m 7} g_{m 10}}{g_{m 7} C_{\pi 10}+g_{m 10} C_{\pi 7}} \\
p_{3}=-\frac{g_{m 8} g_{m 10}\left[g_{m 8}\left(R_{1}+R_{2}\right)\left(g_{m 12} R_{4}\left(g_{m 1}+g_{d s 1}\right)+g_{m 1}\right)+g_{m 1} g_{m 12} R_{4}\right]}{\left[g_{m 8} g_{m 9}\left(R_{1}+R_{2}\right)\left(g_{m 12} R_{4}\left(g_{m 1}+g_{d s 1}\right)+g_{m 1}\right)+g_{m 1} g_{m 12} R_{4}\left(g_{m 8}+g_{m 9}\right)\right] C_{\pi 10}} \\
p_{4}=-\frac{\left.g_{m 10}\left[g_{m 12}\left(R_{1}+R_{2}\right) R_{4}\left(g_{m 1}+g_{d s 1}\right)+g_{m 1} R_{2}\right)\right]}{g_{m 8} g_{m 9} g_{m 12} R_{4}\left(g_{m 1}\left(R_{1}+R_{2}\right)+g_{d s 1} R_{1}\right) C_{\pi 10}}
\end{gathered}
$$

\section{Conclusion}

Poles and zeros include essential information for circuit design. Therefore, the extraction of symbolic expressions for poles and zeros has attracted the attention of symbolic analysis researchers. First reported techniques imitated the usual techniques applied for hand analysis: elimination of unimportant terms in symbolic network functions (considered simplification after generation techniques in the classification used in this chapter), application of root splitting techniques and extraction of roots of low-order symbolic polynomials. These techniques also inherited the exponential growth of symbolic results with the circuit size. Therefore, more recent analysis techniques have incorporated simplification before and during generation techniques. An essential component of the symbolic pole/zero extraction technique is the error control mechanism. Some techniques have used the same approximation techniques used for symbolic network equations, based on the control of magnitude and phase errors. However, a well-controlled magnitude and phase error does not necessarily imply accurate symbolic pole-zero solutions. Therefore, more recent techniques have focused on the development of analysis techniques together with error control mechanisms specifically devoted to guarantee the desired accuracy on symbolic pole/zero expressions.

\section{Acknowledgement}

The preparation of this chapter has been partially supported by the TIC-2532 Project, funded by Consejería de Innovación, Ciencia y Empresa, Junta de Andalucía and by the Project TEC2010-14825/MIC funded by the Spanish Ministry of Science and Innovation with support from ERDF. Dr. C. Sánchez-López thanks the support of the JAE-Doc program of CSIC, co-funded by FSE.

\section{References}

[1] F.V. Fernández, A. Rodríguez-Vázquez, and J.L. Huertas, “A tool for symbolic analysis of analog integrated circuits including pole/zero extraction", in $10^{\text {th }}$ European Conference on Circuit Theory and Design, 1991, pp. 752-761.

[2] F.V. Fernández, A. Rodríguez-Vázquez and J.L. Huertas, "Interactive AC modelling and characterization of analog circuits via symbolic analysis”, Analog Integrated Circuits and Signal Processing, vol. 1, no. 3, pp. 183-208, Nov. 1991. 
[3] G. Gielen, H. Walscharts and W. Sansen, "ISAAC: a symbolic simulator for analog integrated circuits”, IEEE Journal of Solid-State Circuits, vol. 24, pp. 15871597, Dec. 1989.

[4] G. Nebel, U. Kleine and H. Pfleiderer, "Symbolic pole/zero calculation using SANTAFE”, IEEE Journal of Solid-State Circuits, vol. 30, no. 7, pp. 752-761, July 1995.

[5] J. Vlach and K. Singhal, Computer methods for circuit analysis and design, $2^{\text {nd }}$ ed. Van Nostrand Reinhold, 1993.

[6] G. H. Golub and C. F. Van Loan, Matrix computations, $3^{\text {rd }}$ ed. Johns Hopkins, 1996.

[7] J.-J. Hsu and C. Sechen, "Accurate extraction of simplified symbolic pole/zero expressions for large analog IC's”, in IEEE International Symposium on Circuits and Systems, 1995, pp. 2083-2087.

[8] G. Droge, T. Czysz and E.-H. Horneber, "Symbolic pole and zero estimation for circuit design", in IEEE International Conference on Electronics, Circuits and Systems, 1996, pp. 93-97.

[9] E. Hennig, "Matrix approximation techniques for symbolic extraction of poles and zeros", Analog Integrated Circuits and Signal Processing, vol. 31, pp. 81-100, 2002.

[10] J.-J. Hsu and C. Sechen, "Fully symbolic analysis of large analog integrated circuits”, in IEEE Custom Integrated Circuits Conference, 1994, pp. 457-460.

[11]O. Guerra, J.D. Rodríguez-García, F.V. Fernández and A. Rodríguez-Vázquez, "A symbolic pole/zero extraction methodology based on analysis of circuit timeconstants”, Analog Integrated Circuits and Signal Processing, vol. 31, pp. 101-118, 2002.

[12]S. B. Haley, “The generalized eigenproblem: pole-zero computation”, Proceedings of the IEEE, vol. 76, no. 2, pp. 103-120, Feb. 1988.

[13]S. B. Haley, "Modification-decomposition transformation in analog design", International Journal of Computer Aided VLSI Design, pp. 407-428, 1991. 